\title{
Foraging Optimization Through Nectar Quality Prediction - Evolution of Feeding Strategy by Common Myna for Butea monosperma Lam.
}

\section{Subhamay Bhattacharya ${ }^{1,3}$, Koutilya Bhattacharjee ${ }^{2 *}$, Partha Ganguly ${ }^{2}$, Sayan Gupta ${ }^{2}$, Suman Pal $^{2}$, Sourav Halder ${ }^{2}$ and Apratim Maity}

${ }^{1}$ Department of Zoology, Sarojini Naidu College for Women, India

${ }^{2}$ Department of Zoology, Rama Krishna Mission Vivekananda Centenary College, India

${ }^{3}$ Department of Veterinary Biochemistry, West Bengal University of Animal and Fishery Sciences, Kolkata, India

*Corresponding Author: Koutilya Bhattacharjee, Assistant Professor, Department of Zoology, Ramakrishna Mission Vivekananda Centenary College, India.
Received: October 27, 2021

Published: November 30, 2021

(C) All rights are reserved by Koutilya

Bhattacharjee., et al.

\begin{abstract}
Common Myna (Acridotheres tristis), the most abundant avian omnivore in the Indian Peninsula has a diverse food habit of consuming natural as well as human left over (HLO) foods. However, they have evolved a foraging optimization strategy of consuming nectar of Palash (Butea monosperma) from late winter to spring as primary food and other food types as secondary. Such food shifting did not alter their Protein - Lipid - Carbohydrate requirement. The study also reveals that birds have evolved strategies to identify better nectar variant of Butea monosperma plants and concentrate on those plants only while neglecting the less profitable one. Seasonal body weight comparison in humanized and natural habitat also correlates with their food choice. From the aforesaid observations it can be assumed that feeding optimization of Common Myna has definite link to its pre-breeding season preparation.

Keywords: Foraging Optimization; Nectar Quality; Common Myna; Butea monosperma; Breeding Strategy
\end{abstract}

\section{Introduction}

The Indian Myna or Common Myna (Acridothere stristis) is an extremely familiar, omnivorous and a hole-nester species [1]. They show high adaptability in both its natural habitat i.e. deciduous forest as well as anthropoid environment like cities, towns and other human settlements. Common Myna (CM henceforth) principally breeds from April to July, which might extend up to September [1,2]. During breeding season CM feeds mostly on insects, fruits as well as seeds [3]. Although, during late winter and spring which prevails between late February to March locally, in Indian subcontinental climate, CM feeds on nectar of Palas [3]. Around same time flowers burst open in great clusters of vermilion in February-March and are fertilized by CM and others yielding pod ripping from March to June [4]. CM has evolved to coincide its breeding with post flowering and by mid-April they lay their eggs, which is followed by incubation and brooding. In general, avian breed- ing preparations usually include body weight gain and successful choosing and defending of better nesting sites through high competition. Also, defending nests from both intra and inter-specific competition and nest activity for incubating eggs all these behaviours are both time and energy consuming [5]. Therefore, pre-breeding season food choice must be highly profitable one so that feeding and foraging time can be remarkably reduced.

The nectar components of Palas have been analyzed and its pharmacological properties has been thoroughly investigated as well as documented by several workers [6-8]. Flowers with high sugar concentration of nectar has reportedly been noticed to be the first choice amongst nectarivorous birds [9]. Surprisingly, Sunbirds and Humming birds show concentration and temperature specific sugar intake [9], thus sugar composition plays a significant role in food choice. 
Foraging Optimization Through Nectar Quality Prediction - Evolution of Feeding Strategy by Common Myna for Butea monosperma Lam.

The present study focuses on a specific behaviour of CM. While feeding on nectar, the birds specifically prefer some individual Palas plants neglecting the others. Interestingly, all birds concentrate on those individual plants (fed type), while the other plants remain vacant (non-fed type). Such behaviour has been found on each and every sampling period and location. But, the reason behind this preferential choice has no previous report and/or explanatory work. Therefore, this study of the correlation between quality of nectar and optimized feeding by CM is a worthy endeavour.

\section{Materials and Methods}

Study area

Two types of habitats of CM were chosen for the study, one natural without regular human intervention and others with regular human activity. The natural vegetation at the south eastern side of Maithon Reservoir (23047'16"N, 86 ${ }^{\circ} 49^{\prime} 29^{\prime \prime}$ ) was chosen as the Natural Habitat (i.e. NH) whereas areas adjoining Durganagar Railway Station $\left(22^{\circ} 65^{\prime} 05^{\prime \prime} \mathrm{N}, 88^{\circ} 41^{\prime} 09^{\prime \prime} \mathrm{E}\right)$ and adjoining areas of Jadavpur University, Salt Lake Campus $\left(22^{\circ} 56^{\prime} 05^{\prime \prime} \mathrm{N}, 88^{\circ} 41^{\prime} 35^{\prime \prime} \mathrm{E}\right)$, as well as Palta Park area $\left(22^{\circ} 47^{\prime} 27^{\prime \prime}\right.$, $88^{\circ} 21^{\prime} 09^{\prime \prime}$ ) were selected as the Humanized Habitat (i.e. HH). Study areas are depicted in figure 1 . The study period was 5 consecutive years from February 2014 to April 2019.

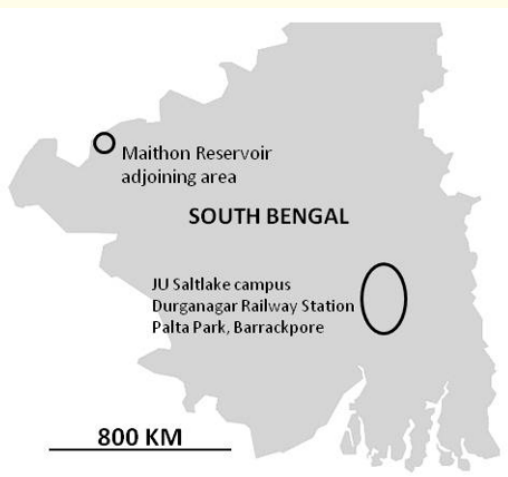

Figure 1: Location of the study area in Eastern India. Small circle is the natural habitat and the large circle encompasses three different humanized habitats.

\section{Body weight of common myna}

Comparative body weight measurement was done using a static population approach. Randomly 50 birds were captured very carefully using fine nylon nets to avoid any harm to the animals for each season for every year for the entire study period. Body weight of each bird was measured and the birds were released soon after. Birds were sampled from two separate regions i.e. natural habitat and humanized habitats, in four different seasons i.e. non-breeding (NB), pre-breeding (PB), breeding (B) and post-breeding (PoB). The pre-breeding season overlaps with the flowering season of $\mathrm{Pa}-$ las. Body weight was measured using WENSER electronic balance (model no. PGB301; Wenser Weighing Scales Limited, Chennai, India) keeping the bird in a transparent plastic bottle.

\section{Behaviour study}

Daily activities of CM populations in natural habitat as well as in different humanized habitats were recorded according following Kemp's methodology [10]. Daily activities were recorded in from 06.00 hours to 17.00 hours, during the study for 21 consecutive days. Records were separately taken for both flowering seasons as well as non-flowering seasons of Palas. Using the foraging behaviour data ethograms were constructed for both flowering and nonflowering as well as for natural and humanized habitats based on previously reported methodology [11-14].

\section{Nectar collection}

Butea monosperma bears flowers that have their nectar concealed [15]. Nectar from fresh unpecked flowers of Butea monosperma were collected in the morning (between 07.00 hrs to 10.00 hrs IST) and also in the afternoon (between $15.00 \mathrm{hrs}$ to $16.00 \mathrm{hrs}$ local time) using sterile disposable syringe and no. 22 needle and were then transferred to sterile micro-centrifuge tubes. These two time periods were chosen after standardization of highest nectar secretion timing. While studying feeding of CM on Palas, it was observed that most of the birds were concentrating on specific individual plants, neglecting others. Thus, plants were separated into two groups fed type (birds feeding on them) and non-fed type (birds not feeding on them). Such behaviour has been found on each and every sampling. The samples were kept on ice and transferred to local field station for further analysis.

\section{Dye isolation and quantitation}

Colour dye extraction was done following Pandit, 2016 [16] with some modifications. Flowers from fed-type and nonfed-type plants were separately collected. Fresh collected flowers were washed carefully in deinonized double distilled water and shed dried. Briefly, petals were separated from $100 \mathrm{gm}$ of shed dried fresh flowers and were used for total dye extraction in soxhlet apparatus using $50 \% \mathrm{v} / \mathrm{v}$ aquas solution of methanol. Temparature of the soxhlet apparatus was maintained at $30^{\circ} \mathrm{C}$ constantly through the extraction. The extracted solution was then air dried at $35^{\circ} \mathrm{C}$ in controlled condition trey dryer. Spectrophotometric quantitation of total dye content from both fed-type and nonfed-type flowers were done following the method described elsewhere [16]. 


\section{Biochemical analysis}

Nectar from individual flowers was assessed for either Total Reducing Sugars (TRS) or Total Protein (TP) or Total Lipid (TL). Total reducing sugar (TRS) was determined according to di-nitro salicylic acid (DNS) method [17]. Nectar protein contents were quantitated using Bio-Rad Protein Assay kits following Bradford's principles of protein assay [18]. TL content were estimated according to the method of Ito and Kaneko [19].

\section{Statistical analysis}

All statistical analyses were done using Statistica version 13.0.

\section{Results and Discussions}

Body weight and feeding time comparison

It was observed that body weight rises significantly in prebreeding (PB) season which sharply falls during breeding season as they remain engage in reproduction followed by egg defence and egg incubation for a significant time of the day (Figure 2).

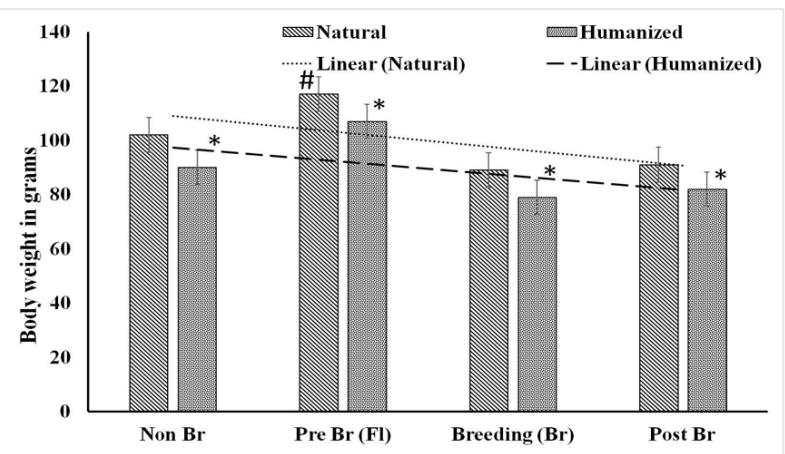

Linear (Natural) $y=-6.1 x+115 ; \quad$ Linear $($ Humanized) $y=-5.2 x+102.5$

Figure 1: Graphical representation of seasonal variation of body weight of Common Myna in natural as well as humanized habitats. Body weight of CM ranges from $80 \mathrm{~g}$ to $120 \mathrm{~g}$. Average body weight values of CM in non-breeding (non br), pre-breeding (pre br), breeding (br) and post-breeding (post br) seasons are 102g,

$117 \mathrm{~g} .89 \mathrm{~g}$ and $91 \mathrm{~g}$ in natural habitats whereas for humanized habitats such values are $90 \mathrm{~g}, 107 \mathrm{~g}, 79 \mathrm{~g}$ and $82 \mathrm{~g}$ respectively. Average values obtained from 50 birds per season for 5 seasons. The differences between natural and humanized habitat values for each season are significant at 0.05 level and the difference of pre breeding values with other seasonal values are also significant at 0.05 level.

Pre Br (Fl)- Pre-Breeding Flowering, *- Denotes Statistical Significance
This reduction remains till the offsprings grew up and the bird enters the non-breeding season when they primarily concentrate on feeding. The differences of body weight between natural and humanized habitats are significant at $\mathrm{p}=0.05$ among all groups as well. The pre-breeding weight gain among birds is already known as birds feed more and more during that season. But CM achieves such gain with evolutionary smartness. As the pre-breeding season coincides with flowering season of Palas, CM gets an abundantly available high-energy food by opting to them effectively reducing feeding time (Figure 3a). In flowering seasons, foraging on nectar comprises more than $60 \%$ of total foraging time in the natural habitat. In flowering season the foraging time decreases highly even in natural habitat, from 510 mins/day of non-flowering season to 380 mins/day. Such big time saving of more than 2 hours (130 minutes) per day allows the bird for searching nesting sites and building nests.

It is obvious that natural habitat contain higher number of $\mathrm{Pa}$ las plants that can support the need of CM population there but in case of humanized habitat where number of Palas plant is much reduced the birds require to forage (Figure $3 \mathrm{~b}$ ) in the fields for other food sources even in the flowering season. As it is shown CM spends a major time both in the morning and afternoon hours for foraging in field. Therefore, numbers of nests per plant are reduced in humanized habitats (data not shown). Feeding time also significantly increases in the humanized habitat from 510 minutes/day in flowering season to 580 minutes/day in humanized habitats. Therefore nesting behaviour in humanized habitat definitely compromises feeding thus reducing average body weight (Figure 3).

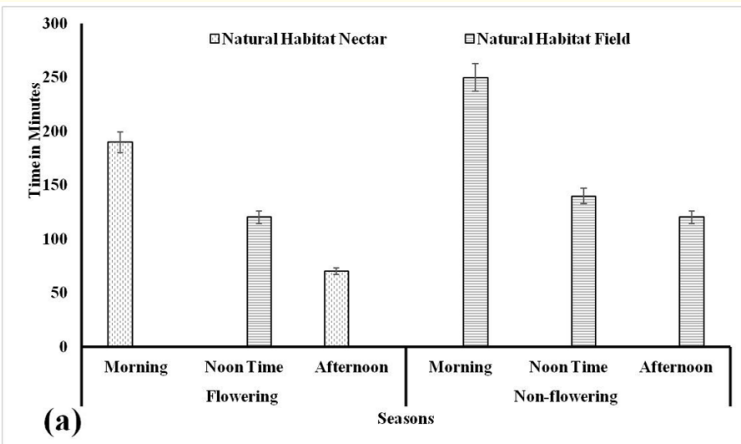




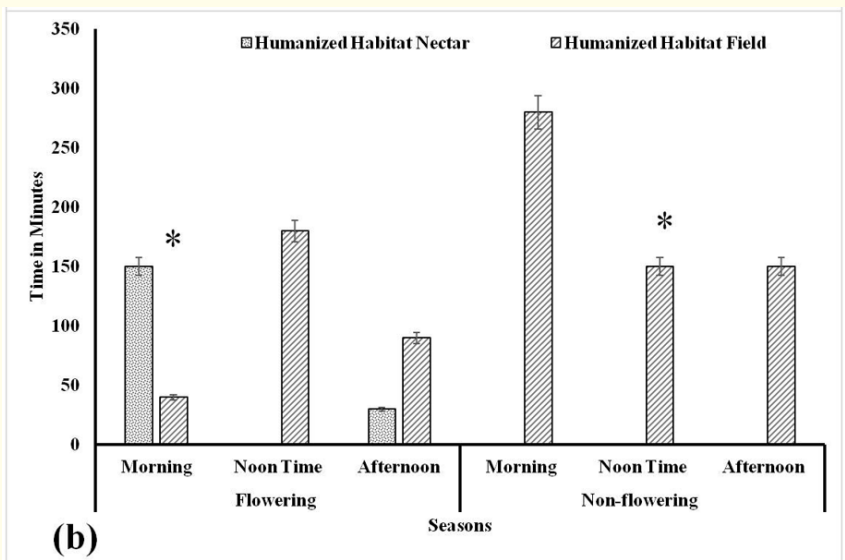

Figure 3: Comparative foraging timing of CM in flowering and non-flowering season between natural and humanized habitats. In flowering season, the bird spends on average 380 minutes/day and 490 minutes/day in natural and humanized habitats respectively. In humanized habitats the daily foraging time is very high therefore nest building compromises feeding.

*- Denotes Statistical Significance.

\section{Colour dye content of flowers}

Fresh shed-dried flowers weigh in the range of 3.25 to 3.65 grams. Consistently, petal weights of every flower bear nearly $75 \%$ of the flower weight on average. One hundred gms of each type flower yielded 0.323 gms to 0.329 gms (Figure 4) of pigment from fed-type and non fed-type flowers respectively.

Results are average of 10 replicates. Flowers from each plant types were collected twice per flowering season for 5 consecutive years. Such amount was a little higher from the values reported by Pandit, 2016 [16]. However, there was no significant difference of flower pigment content between fed-type and non fed-type flower. Such observation leads to the point that pigment content may not be the visual cue for the birds to identify more profitable flower.

\section{Biochemical analysis of nectar}

Total sugar content of fed-type nectar was remarkably higher that that collected from non fed-type Palas flowers (Figure 5a). Fed-type nectar contain 33\% more sugar than non fed-type nectar. Besides, $65 \%$ of total sugar in fed-type nectar is in reducing sugar form where as such rate is less than $50 \%$ in non fed-type flower.

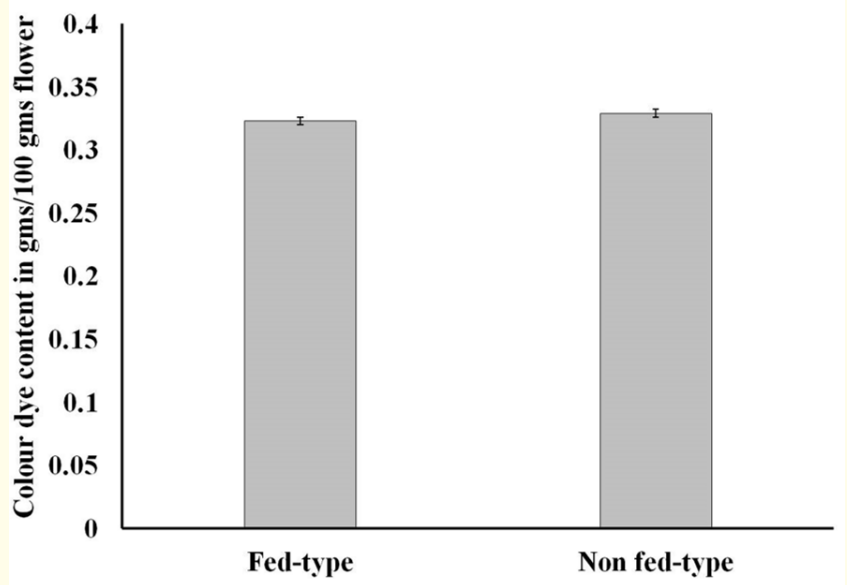

Figure 4: Colour dye content of fed-type and non fed-type flowers. The content is more or less equal per $100 \mathrm{gms}$ of petal for both type of flowers.

There is no significant difference of protein and lipid contents between fed-type and non-fed-type nectar (Figure 5b).

Therefore, choosing the more profitable type (produced by the fed-type plants flowers) of nectar will definitely reduce their foraging time as depicted in figure 3 , which can be used for nest preparation related activities in this pre-breeding season. The present authors also found that fruit production is obvious among fed-type plants whereas non fed-type plants may or may not lead to fertilization due to lack of big pollinators (data not shown). Such difference of nectar quality is definitely recognized by the birds somehow.

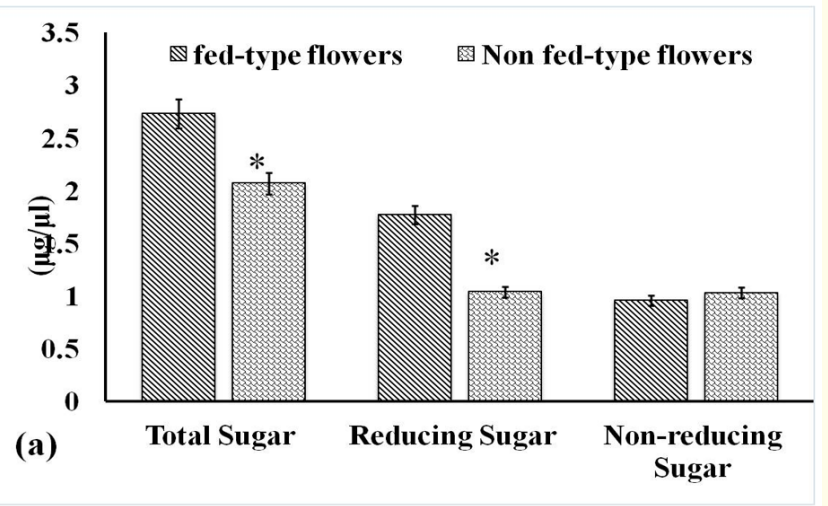




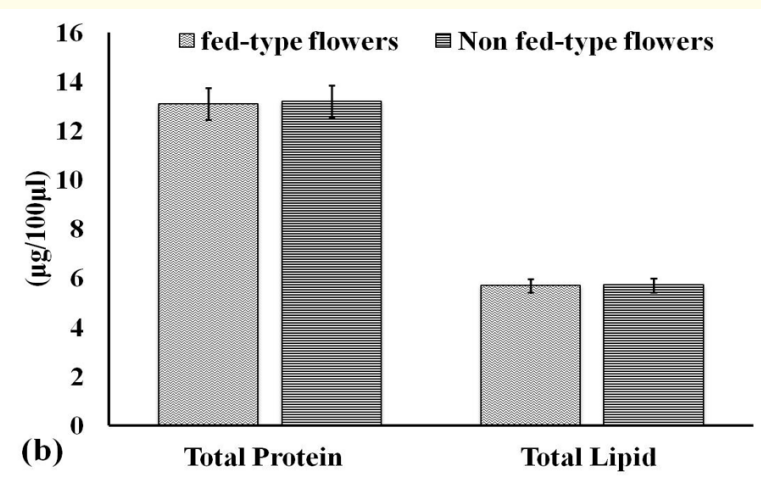

Figure 5: Comparative carbohydrate - protein - lipid contents of nectar from fed-type and non fed-type flowers. Total sugar and reducing sugar content of fed-type nectar are much higher than those of non fed-type nectar and the differences are significant at $\mathrm{p}^{*}=0.05$. However, total protein and total lipid content are more or less similar among the two nectar types.

\section{Conclusion}

Preferential choice of fed-type nectar as food has helped CM in several ways. The pre-breeding birds reduce both time and energy of travelling and handling. Correlating this fact with the ethogram data reveals that energy expenditure in foraging and roaming is significantly lesser in the flowering seasons. Lesser activity with higher food gain is the basic way to gain body weight and CM has optimized that in an evolutionary way. Such behaviour is highly helpful for breeding preparation.

Present study estimated total pigment content of both fed-type and non fed-type flowers as same however the authors believe that further detailed analysis is important and such study is currently being undertaken by the authors to identify individual pigment component types and their quantity per flower.

Difference in nectar quality can be due two different plant proteins. First the "Sugar Transporters" that allows release of sucrose from nectary cells into the floral cup and the second one is the "Cell wall Invertase" which breaks the sucrose into its reducing components glucose and fructose. There might be any genetic mechanism involving either genetic polymorphism or elements of differential gene expression or may be both underlying such difference of protein function. Thorough methodical further analysis in this regard will definitely help in identification of the better variant.
In urban or semi urban areas, natural food and potential nesting site of CM is already very less and the birds are concentrating more on human left overs as food and vacant building places as nesting sites. Such urbanization of wildlife and intrusion of wildlife in human places is compromising the ecological balance of urban plants and animals affecting human civilization in many ways. The authors have also observed that all fed-type flowers produced fruit within two months every year whereas non fed-type flowers produced fruits at least three and half months on average tough about $20 \%$ to $30 \%$ of the plants yielded fruits (supplementary table). Therefore, preferential planned forestry of fed-type Palas variants will definitely increase natural food of CM as well as tentative natural nesting sites. Thus, such plantation may be great help in reducing wildlife intrusion to human civilization restoring the ecological balance.

\section{Acknowledgement}

This work has partly been done with financial support from Inhouse Research Grant vide sanction No: SNCW/ 231/10-2016 dated 08.12.2016 of Sarojini Naidu College for Women, Kolkata, India.

\section{Conflict of Interests}

The authors declare no conflict of interests regarding this manuscript.

\section{Bibliography}

1. Mahabal A. "Activity-time budget of Indian Myna Acridotheres tristis (Linnaeus) during the breeding season". Journal of the Bombay Natural History Society 90.1 (1991): 96-97.

2. Ali Salim. "The book of Indian birds. Bombay". Natural History Society: Bombay (1996).

3. Sengupta S. "Food and feeding ecology of the common myna Acridotheres tristis". Proceedings of the Indian National Science Academy 42.6 (1976).

4. Sahni KC. "The book of Indian trees". Oxford University Press (1999).

5. Siddique M., et al. "Breeding Behaviour of Common Myna (Acrjdotheres Tristis)". The Pakistan Journal of Agricultural Sciences 30.4 (1993).

6. Sindhia VR and R Bairwa. "Plant review: Butea monosperma". International journal of pharmaceutical and clinical research 2.2 (2010): 90-94. 
7. Prachayasittikul Supaluk., et al. "Antimicrobial and antioxidative activities of bioactive constituents from Hydnophytum formicarum Jack". Molecules 13.4 (2008): 904-921.

8. Jamkhande PG., et al. "In vitro antioxidant activity of Butea monosperma flowers fractions". International Journal of Drug Development and Research 5 (2013): 245-255.

9. Schondube Jorge E., and Carlos Martínez Del Rio. “Concentration-dependent sugar preferences in nectar-feeding birds: mechanisms and consequences". Functional Ecology 17.4 (2003): 445-453.

10. Kishida Yuriko and Coral Kemp. "A measure of engagement for children with intellectual disabilities in early childhood settings: A preliminary study". Journal of Intellectual and Developmental Disability 31.2 (2006): 101-114.

11. Barnard CJ. "Flock feeding and time budgets in the house sparrow (Passer domestic us L.)”. Animal behaviour 28.1 (1980): 295-309.

12. Wolf Larry L., et al. "Foraging efficiencies and time budgets in nectar-feeding birds". Ecology 56.1 (1975): 117-128.

13. Maxson Stephen J and Lewis W Oring. "Breeding season time and energy budgets of the polyandrous spotted sandpiper". Behaviour 74.3-4 (1980): 200-263.

14. Fleming PA., et al. "Concentration and temperature effects on sugar intake and preferences in a sunbird and a hummingbird". Functional Ecology 18.2 (2004): 223-232.

15. Tandon Rajesh KR., et al. "Reproductive biology of Butea monosperma (Fabaceae)”. Annals of Botany 92.5 (2003): 715-723.

16. Pandit PS., et al. "Colour Extraction from Butea monosperma (palash) Flowers”. Journal of Tree Sciences 35.2 (2016): 19-22.

17. Kim Yong-gyun. " $\beta$-glucanase Bacillus sp. CSB34: Screening, Production, Partial Purification, and Biochemical Characterization”. Diss. Chosun University Graduate School (2017).

18. Kruger Nicholas J. "The Bradford method for protein quantitation". The protein protocols handbook (2009): 17-24.

19. Itoh Toshihiro and Hiroshi Kaneko. "Yeast lipids in species variation. I. A simple method for estimating the cellular lipids". Journal of Japan Oil Chemists' Society 23.6 (1974): 350-354.
Volume 3 Issue 12 December 2021 (C) All rights are reserved by Koutilya Bhattacharjee., et al. 